\title{
A Gender Recognition System from Facial Image
}

\author{
Md. Nurul Ahad Tawhid \\ Institute of Information Technology \\ University of Dhaka \\ Dhaka, Bangladesh
}

\author{
Emon Kumar Dey \\ Institute of Information Technology \\ University of Dhaka \\ Dhaka, Bangladesh
}

\begin{abstract}
Automatic gender classification from facial image has become an attractive research area in the field of machine learning. Various methods have already been proposed for gender recognition in both controlled and uncontrolled situations. Problem arises in uncontrolled situation when there are high rate of noises, lack of illumination etc. To mitigate the problems, we have proposed a framework where we applied a pre-processing to enhance the images using $\mathrm{Bi}$ lateral Histogram Equalization (BHEP) algorithm and applied the proposed framework in LFW, Adience and color FERET dataset yielding $94.29 \%, 84.86 \%$ and $98.30 \%$ accuracies. Confusion matrix, Precision, Recall, F-measure, True Positive Rate (TPR), True Negative Rate (TNR) etc. also shows that our proposed method performs better than the existing state of the arts.
\end{abstract}

\section{Keywords}

Gender recognition, image enhancement, BHEP, image preprocessing, image enhancement, feature extraction

\section{INTRODUCTION}

Gender recognition from face images is one of the fundamental research area in computer vision. Automated gender recognition is important in many application area such as human computer interaction, biometric, surveillance, demographic statistics etc. Human face contains important visual information for gender perception. It is challenging for a machine to identify these visual information which separate male faces from female faces. Researches are going on so that a machine can achieve human level accuracy.

Various methods have been proposed for classifying gender from several controlled and uncontrolled dataset. It is more challenging in uncontrolled situations. Beside these some face images are so confusing, in most of the time a human is also fail to detect the gender from the image. So there is a wide scope for improving the performances of gender recognition approaches.

Various neural network approaches were employed in early 1990s for gender recognitions. Using a fully connected two layer network namely SEXNET, Golomb et. al [7] achieved $91.9 \%$ accuracy using 90 facial images in a controlled situation. Baluja et al. [4] proposed a method using boosting pixel comparison of face images. They used FERET database [20] and Support Vector Machine (SVM) for classification on images of $20 \times 20$ pixels. Makinen et al. [16] also proposed a method by considering FERET database. Images of FERET dataset are captured under controlled environment such as; frontal faces, consistent lighting etc. In real time scenario it is necessary to detect gender in uncontrolled situation which is challenging. Concerning this, Shakhnarovich et al. [24] took over 3,500 facial images from websites which are captured on uncontrolled situations. They applied Haar-like features extraction method. Using Adaboost and SVM classifier they got $79 \%$ and $75.5 \%$ accuracies respectively. Labeled Face in the Wild (LFW) dataset [10] containing more than 13,000 face images was developed for studying the problem of unconstrained face recognition. Caifeng Shan [25] applied his gender recognition method in 7,443 images from LFW dataset and found $94.81 \%$ accuracy. He applied boosted Local Binary Pattern Histogram as features and SVM for classification by not considering 5,790 difficult images of LFW dataset.

Numerous local feature descriptors have been proposed in the field of computer vision. Local Binary Patterns (LBP) [1] and its variants such as Local Ternary Patterns (LTP) [27], Local Gradient Pattern (LGP) [12], Local Derivative Pattern (LDP) [31], Local Directional Texture Pattern (LDTP) [23] , Completed LBP (CLBP) [9] etc. have been proposed recently. CENsus TRansform hISTogram (CENTRIST) [29] is another visual texture descriptor proposed for scene categorization which is very similar to LBP and gained popularity because of their computational simplicity. These descriptors are also now using in the field of face and gender recognition. Though, these descriptors shows good accuracies in different controlled environment, performance are still not satisfactory in uncontrolled scenarios. Recently a texture descriptor Noise Adaptive Binary Pattern (NABP) [21] is proposed which encode the local structure of a face image and intensity variation based on adaptive threshold. Using Adaboost classifier the authors achieved 92.74\% accuracy on LFW dataset containing more than 13,000 images with all uncontrolled difficult images. Rahman et. al [22] proposed a gender recognition approach with embedded preprocessing. They applied Adaptive Gamma Correction with Weighted Distribution (AGCWD) [11] to reduce the noises on original images and found better result in LFW dataset using different feature extraction methods. Zhang et al [32] applied Fisher Vector encoding [26] for feature extraction. They used PCA to reduce the dimensionality and by applying linear SVM classifier they achieved $92.5 \%$ and $92.3 \%$ accuracies for aligned and misaligned images of LFW dataset. Dey et. al [5] proposed two new feature extraction methods based on CENTRIST namely cCENTRIST and tCENTRIST. Although they proposed these two methods for garments texture classification, these two also can be used in different facial image analysis research. Azarmehr et al [3] applied Multi-scale Local Binary Patterns (MSLBP) proposed by [18] for gender recognition. They proposed to apply a Bilateral filtering approach in preprocessing to 
suppress the noises. Using SVM classifier they achieved $79.88 \%$ accuracy on Adience dataset. Table 11 summarizes several gender recognition methods and their accuracy on different databases. Recently Deep Convolutional Neural Network (CNN) are being applied on face and gender recognition [15, 33]. Different CNN architectures have already been proposed for this purpose. In this paper we will propose an approach for classifying gender from facial images using the feature extraction method tCENTRIST. The contribution of this paper are as follows:

(1) We have introduced an automated system for gender recognition from facial images.

(2) For enhancing the input image, we have used Bilateral Histogram Equalization (BHEP) [2]

(3) We have adopted tCENTRIST for extracting features from the input images.

The rest of the paper is organized as follows: Section 2 describes the background studies, section 3 describes the proposed system architecture of gender detection, section 4 describes the experimental results and finally section 5 concludes the paper.

\section{BACKGROUND STUDIES}

Different methods have been proposed for extracting features from face images and to train the system for identifying gender. We have experimented on different feature extraction methods and adopted tCENTRIST for our proposed system. In the following subsections we will briefly describe some necessary topics related to our proposed system.

\subsection{CENTRIST}

Based on the concept of Census Transform (CT) by R. Zabih et al. [30], J. Wu et al. proposed a non-parametric local transform technique named CENTRIST [29]. To capture the image structure, it produces an eight bit string (CT values) for a pixel by comparing its intensity value with eight neighbouring pixels. The main difference with LBP is that LBP performs interpolation while considering the corner pixels but CENTRIST considers the corner pixel as is. An example of CT calculation is given in Figure 2.1.

CENTRIST uses histogram of the CT values of image blocks for an image to capture both the local and global information. They also used Spatial Pyramid Matching (SPM) [14] scheme based spatial representation to capture the global structure of an image. That scheme divides an image into sub regions and integrate correspondence result of those regions, which improves recognition.

\section{2 tCENTRIST}

Ternary CENTRIST (tCENTRIST) is a fusion of LTP and CENTRIST. It uses LTP in place of LBP of CENTRIST. To capture the global structure tCENTRIST uses spatial representation based on SPM. For each block of Spatial Pyramid (SP), tCENTRIST calculates Local Ternary Pattern (LTP) and two histograms are constructed - one for upper code and another for lower code of LTP. Those two histograms are then concatenated to build a single histogram. Finally, histograms for all blocks are combined and Principal Component Analysis (PCA) is applied for dimension reduction which gives the final feature vector for an image. Algorithm 1 describes this process.

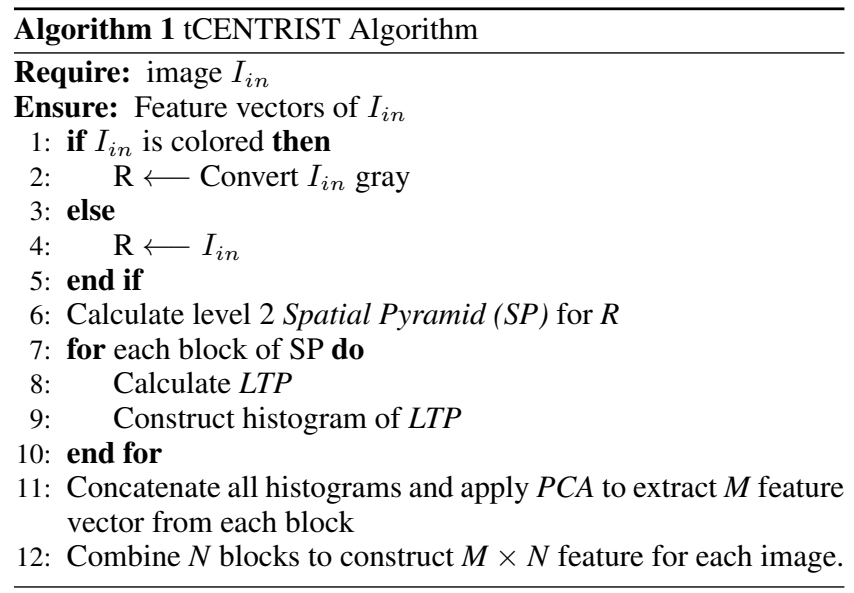

\section{PROPOSED METHOD}

High performance gender recognition methods depend on face detection, facial image preprocessing, feature extraction and finally classification[17]. We have used LFW, audience and color FERET dataset in this research where LFW face images are $100 \times 100$ size, audience face images are $816 \times 816$ size and color FERET face images are $150 \times 150$ size. Before extracting features we preprocessed the training and testing images by using Bilateral Histogram Equalization [2] for enhancing the images and then tCENTRIST is used for extracting the prominent features. Extracted features from the enhanced images are then used for training the system. Linear SVM classifier has been used for classification purpose.

\subsection{Image Enhancement}

Several distinctive properties such as line, corners, spots, edges can be used as features for classifying face images. To clearly visualize the properties, several image enhancement technique can be used. Histogram Equalization (HE) [8], Brightness Preserving Bi-Histogram Equalization (BBHE) [13], Dualistic SubImage Histogram Equalization (DSIHE) [28], Recursively Separated and Weighted Histogram Equalization (RSWHE) [13], Adaptive Gamma Correction with Weighted Distribution (AGCWD) [11] etc. are widely used in this area. Histogram Equalization is a popular image enhancement technique but sometimes it over enhance the image and performance degrades in some classifications. BBHE was proposed to mitigate the mean shift problem of (HE) but BBHE fails in the situation for non-symmetric distribution. ACGWD transform each and every pixel of an image based on the highest pixel intensity in the histogram. So, over enhancement does not occur but it fails to show better result in case of law contrast input image. We have used Bilateral Histogram Equalization with Preprocessing (BHEP) [2] for enhancing the input image proposed by Feroz et. al. BHEP shows better results on low contrast images. It reduce the artifacts of the outliers. They choose harmonic mean to divide the image into two parts for enhancing the image and explained the reason behind choosing the harmonic mean for dividing the image into two parts with proper example. Algorithm 2 shows the detail procedure of BHEP.

\subsection{Feature Extraction and dimension reduction}

After enhancing the input image we applied tCENTRIST to extract features from the face images described on section 2.2. Original tCENTRIST performed well on garments texture identification [5]. 
Table 1. Several existing gender recognition methods and their accuracy

\begin{tabular}{|c|c|c|c|c|}
\hline Ref & Feature & Dataset & No. of Sample & Accuracy \\
\hline$[7$ & Neural Network & Personal & 90 & $91.90 \%$ \\
\hline$[25$ & Boosted LBP & LFW & 7443 & $94.81 \%$ \\
\hline$[24$ & Haar Features & Web Images & 3500 & $79.00 \%$ \\
\hline 21 & NABP & LFW & 13000 & $92.74 \%$ \\
\hline 32 & \multirow{2}{*}{ Fisher Vector encoding } & LFW & 13233 & $92.50 \%$ \\
\cline { 2 - 5 } & \multirow{2}{*}{ MSLBP } & Color FERET & 1762 & $98.00 \%$ \\
\hline & & Adience & 13649 & $79.88 \%$ \\
\hline
\end{tabular}

\begin{tabular}{|c|c|c|}
\hline 45 & 85 & 76 \\
\hline 51 & 77 & 11 \\
\hline 2 & 10 & 88 \\
\hline
\end{tabular}

\begin{tabular}{|l|l|l|}
\hline 1 & 0 & 1 \\
\hline 1 & & 1 \\
\hline 1 & 1 & 0 \\
\hline
\end{tabular}

\section{(10111110)}
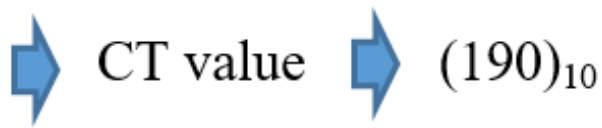

Fig. 1. Example of Census Transform (CT).

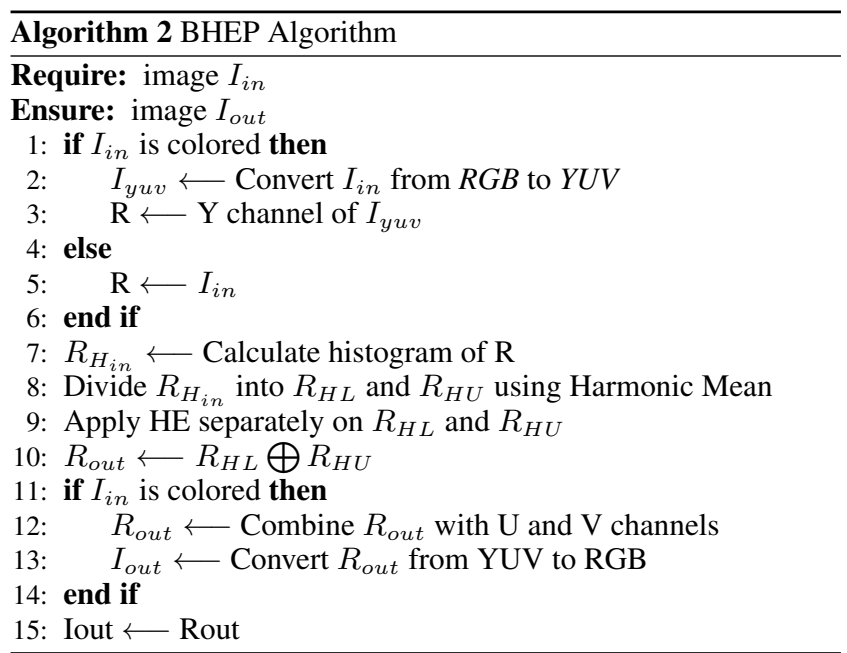

We also appled PCA for reducing the dimensionality of image features. Figure 2 shows the steps of our proposed method.

\subsection{Classifier Training}

After extracting features from the enhanced input image a classifier is used for making decision. In this system we have used Support Vector Machine (SVM) for classifying the features of input images because of its wide applicability for classification and high performance.

\section{EXPERIMENTAL SETUP}

In this section we will firstly describe about our datasets. We have used three different datasets to evaluate our proposed system. After that experimental results with experimental settings and detail training and testing procedures will be described.

\subsection{Dataset}

In order to evaluate the performance of our proposed method, firstly we have used Labeled Face in the Wild (LFW) dataset [10]. This database contains 13,230 face images of 5,749 different persons. Among them 4,263 are male and 1,486 female. Images of this database is also gender imbalanced containing 10,256 male and 2,976 female samples. All the images were collected from web which are captured in uncontrolled situations. Some sample images from LFW dataset are given in Figure 3.

We have also used a relatively newly resealed adience dataset [6] to evaluate the result of our proposed method. The original dataset contains 26,580 face images of 2,284 different person. We have used the front version of this dataset which consist of total 13,649 images where 5772 images are for male face and 7788 are female faces. We considered the gender for all age categories. Table 2 summarize the description of used adience dataset. Figure 4 shows some sample images from adience dataset.

Finally, Color FERET dataset [19] is used for evaluating our system. Color FERET dataset consist of 1199 different subject. Total 2712 facial images are used. All images captured under controlled environment but they are different in ethnicities, facial expression, makeup, illumination condition etc. We have cropped the facial portion using Viola and Jones method. Figure 5 shows some example images from Color FERET dataset.

\subsection{Training and Testing}

We separated male and female faces into different folder. For testing purpose we randomly selected one fifth face images from each categories and rest of the images are used as training images. So, for LFW dataset we randomly selected 8204 male faces and 2381 female faces for training and rest of the face images (2050 male and 595 female faces) for testing. We repeated this process for five times. Again for adience dataset we randomly selected 4617 male and 6230 female faces for training the system and rest of the images (1155 male and 1558 female faces) are used for testing. This process is also repeated for five times. Similarly for color FERET dataset, we randomly selected 1368 male and 802 female faces for training the system and rest of the images (342 male and 200 female 


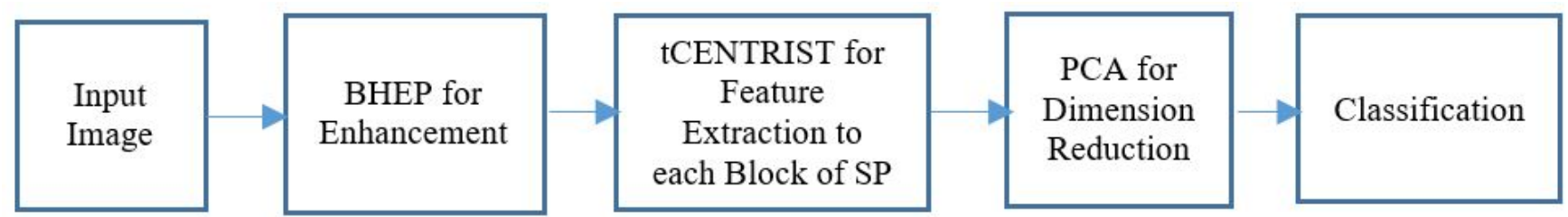

Fig. 2. Proposed method for Gender Identification.

Table 2. Summery of adience dataset [6]

\begin{tabular}{|c|c|c|c|c|c|c|c|c|c|}
\hline \multicolumn{10}{|c|}{ Front version (faces in the range of $\pm 5^{\circ}$ yaw from frontal) } \\
\hline Age Range & $0-2$ & $4-6$ & $8-13$ & $15-20$ & $25-32$ & $38-43$ & $48-53$ & $60-$ & Total \\
\hline Male & 557 & 691 & 738 & 501 & 1,602 & 875 & 273 & 272 & 5,824 \\
\hline Female & 492 & 911 & 956 & 630 & 1,692 & 732 & 295 & 309 & 6,455 \\
\hline T. Gnd. & 1,049 & 1,602 & 1,694 & 1,131 & 3,294 & 1,607 & 568 & 581 & 13,649 \\
\hline Total & 1,843 & 1,602 & 1,700 & 1,132 & 3,335 & 1,607 & 572 & 585 & \\
\hline
\end{tabular}

faces) are used for testing for each time. Support Vector Machine (SVM) is used for classification.

We also considered the overall accuracy, Recall, Precision, FMeasure to calculate the performance which are defined in Equations $1-4$.

$$
\begin{gathered}
\text { Accuracy }=\frac{\text { NumberofCorrectDetection } \times 100}{\text { TotalNumberofTestImages }} \\
\text { Recall }_{i}=\frac{\text { Mii }}{\sum_{j} \text { Mij }} \\
\text { Precision }_{i}=\frac{M i i}{\sum_{j} M j i} \\
F-\text { measure }_{i}=\frac{2 \times \text { Recall }_{i} \times \text { Precision }_{i}}{\text { Recall }_{i}+\text { Precision }_{i}}
\end{gathered}
$$

here, $\mathrm{Mii}=$ Number of correct detection of class $\mathrm{i}$ as class $\mathrm{i} ; \mathrm{Mij}=$ Total number of detection of class $i$ as class $i$ and $j$; Mji $=$ Number of detection of class $j$ detected as class $i$ and $j$. F-measure used for measuring the regression performances which is the harmonic mean of Precision and Recall value. Large value of F-measure indicates higher accuracy.

\section{RESULTS AND DISCUSSION}

We have proposed a system for gender identification from facial images. We applied tCENTRIST for feature extraction and compare the result of the proposed method with other feature extraction techniques. Table 5 shows the confusion matrix of gender detection system without BHEP and Table 5 shows the result using preprocessing. The numbers are putted by making average of total 5 round test.

Table 5 illustrate that our proposed method correctly identifies 482 female and 2008 male among 595 females and 2050 males in LFW database. 113 female and 42 males are misclassified. Again 1355 among 1558 female and 913 among 1155 male faces are correctly identified in Adience dataset. 203 female and 242 male faces are misclassified. In Color FERET dataset our system identified 191 female and 337 male correctly in each rounds among 201 female
Table 5. Percentages of accuracies on different datasets in various situations.

\begin{tabular}{|c|c|c|c|}
\hline \multirow{2}{*}{ Feature extraction using tCENTRIST } & \multicolumn{3}{|c|}{ Accuracy (\%) } \\
\cline { 2 - 4 } & LFW & Adience & Color FERET \\
\hline Proposed method without BHEP & 93.75 & 82.79 & 97.24 \\
\hline Proposed method with BHEP & 94.29 & 84.99 & 98.30 \\
\hline
\end{tabular}

and 342 male face without applying BHEP before feature extraction.

From Table 5 we can see that using BHEP, accuracy in LFW dataset almost remains same whereas Adience dataset shows a signification improvement in terms of accuracy.

From Table 5 it is clear that in each case our proposed method outperform state of the arts. Applying BHEP we found better accuracies on each dataset. Extracting features using tCENTRIST shows 93.75\% accuracy and with BHEP it increases to $94.29 \%$ in LFW dataset which is better than [32]. Again in Adience dataset proposed method shows better accuracy than [3] in both cases.

Our method found $97.24 \%$ and $98.30 \%$ accuracies in Color FERET dataset without using BHEP and with BHEP respectively. Although, in [32], the author claimed $98.0 \%$ accuracy, they used Color FERET 700 dataset which consider only selected 700 images from the original dataset. But we have experimented on all 2712 images of original Color FERET dataset and found better accuracies using our proposed system. We have performed 5 fold cross validation.

Table 6 shows the Precision, Recall and F-measure for LFW, Adience and FERET dataset without pre-processing and extracting features using tCENTRIST. Table 7 shows that the result improves when we applied BHEP before feature extraction.

Figure 6, 7 and 8 show the round wise accuracies in LFW, Audience and color FERET dataset respectively. From these figures it is clear that each round shows better accuracy in each dataset by enhancing images with BHEP before applying tCENTRIST. Figure 9 shows individual Male, Female recognition accuracy on different datasets. Using six different state of the arts feature extraction methods we experimented on LFW, Adience and FERET dataset. Table 8 shows the experimental results on LFW dataset, Table 9 and Table 10 show results for Adience and FERET dataset respectively. It is clear from these three table that tCENTRIST show high accuracies than all other methods. Use of BHEP before feature ex- 


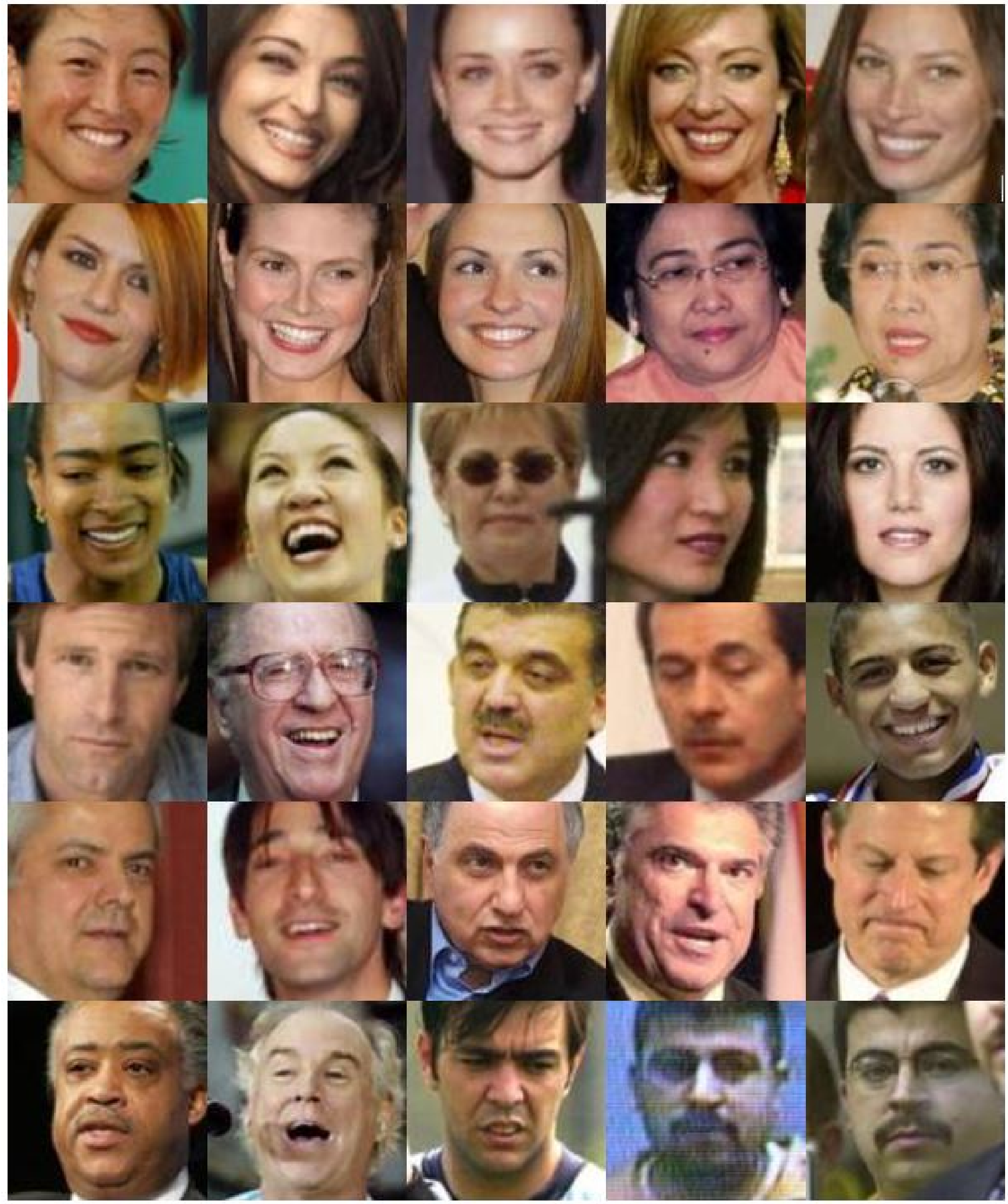

Fig. 3. Sample images form LFW dataset. First three rows are female and last three rows are male faces. 


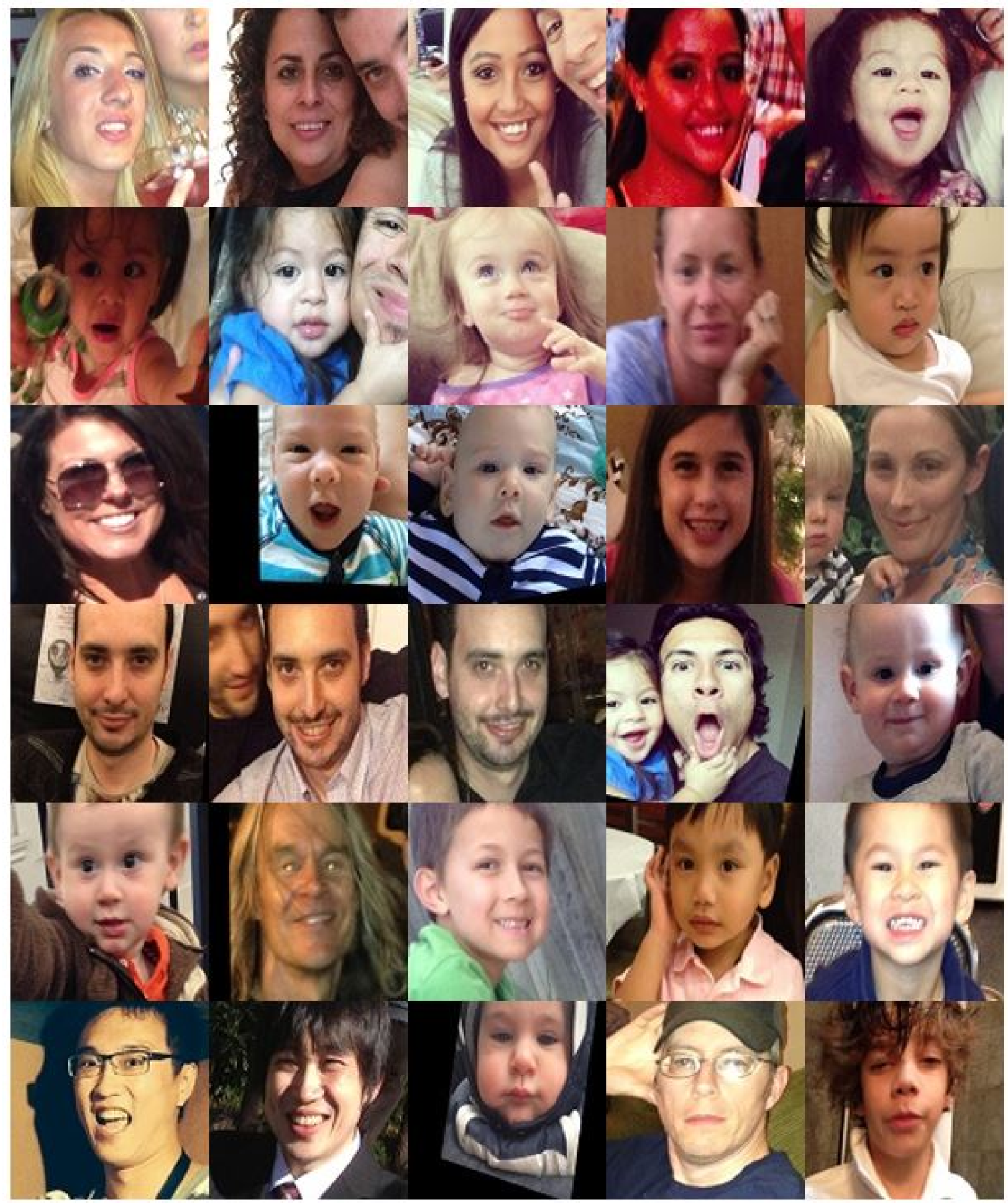

Fig. 4. Sample images form Adience dataset. First three rows are female and last three rows are male faces. 


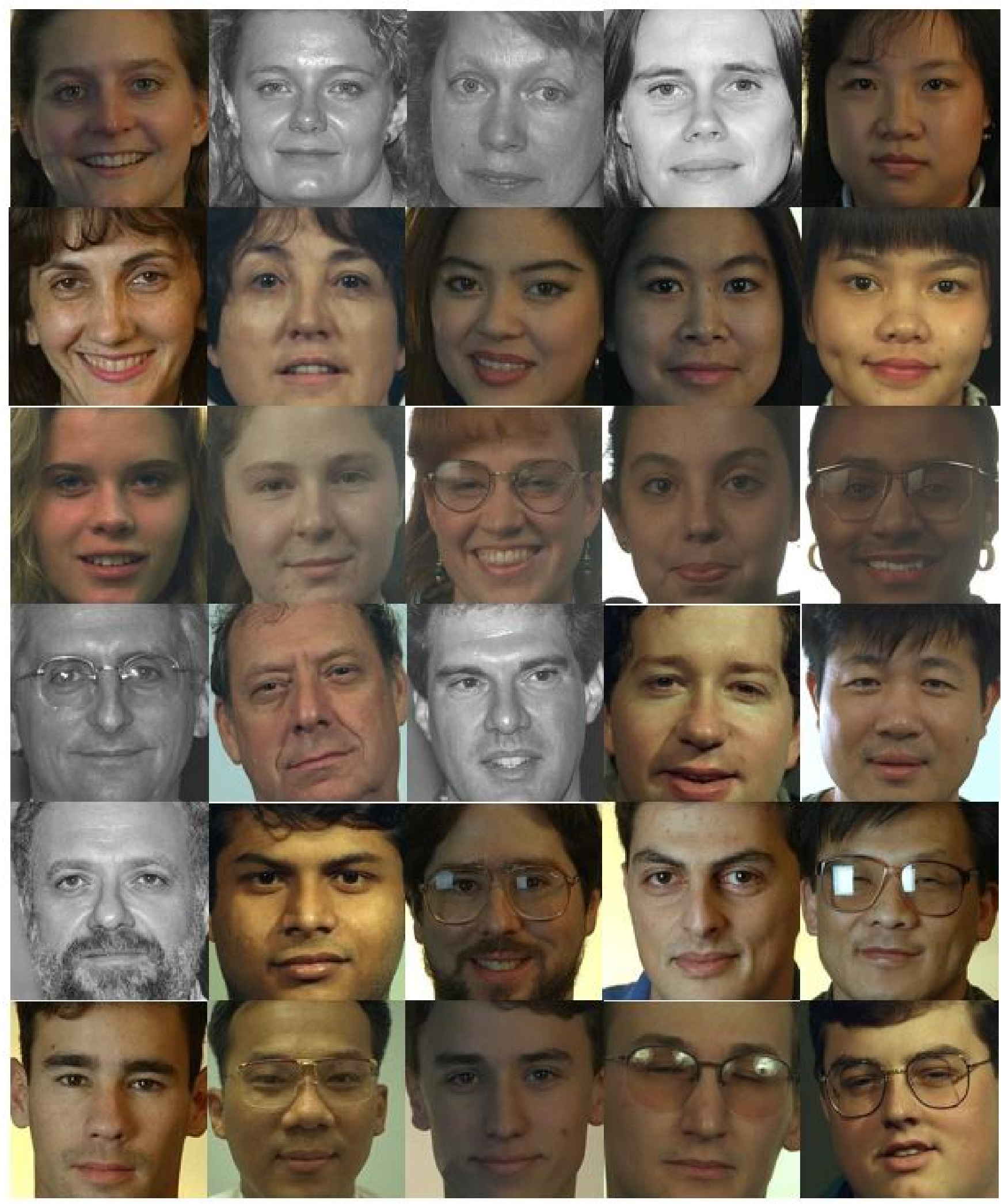

Fig. 5. Sample images from Color FERET dataset. First three rows are female and last three rows are male faces. 
Table 3. Confusion matrix for gender recognition in different dataset without BHEP

\begin{tabular}{|c|c|c|c|c|c|c|}
\hline \multirow{2}{*}{} & \multicolumn{2}{|c|}{ LFW Dataset } & \multicolumn{2}{c|}{ Adience Dataset } & \multicolumn{2}{c|}{ Color FERET Dataset } \\
\cline { 2 - 7 } & Female & Male & Female & Male & Female & Male \\
\hline Female & 482 & 113 & 1355 & 203 & 191 & 10 \\
\hline Male & 42 & 2008 & 242 & 913 & 5 & 337 \\
\hline
\end{tabular}

Table 4. Confusion matrix for gender recognition in different Dataset with BHEP.

\begin{tabular}{|c|c|c|c|c|c|c|}
\hline \multirow{2}{*}{} & \multicolumn{2}{|c|}{ LFW Dataset } & \multicolumn{2}{c|}{ Adience Dataset } & \multicolumn{2}{c|}{ Color FERET Dataset } \\
\cline { 2 - 7 } & Female & Male & Female & Male & Female & Male \\
\hline Female & 484 & 111 & 1375 & 183 & 194 & 7 \\
\hline Male & 40 & 2010 & 225 & 930 & 2 & 340 \\
\hline
\end{tabular}

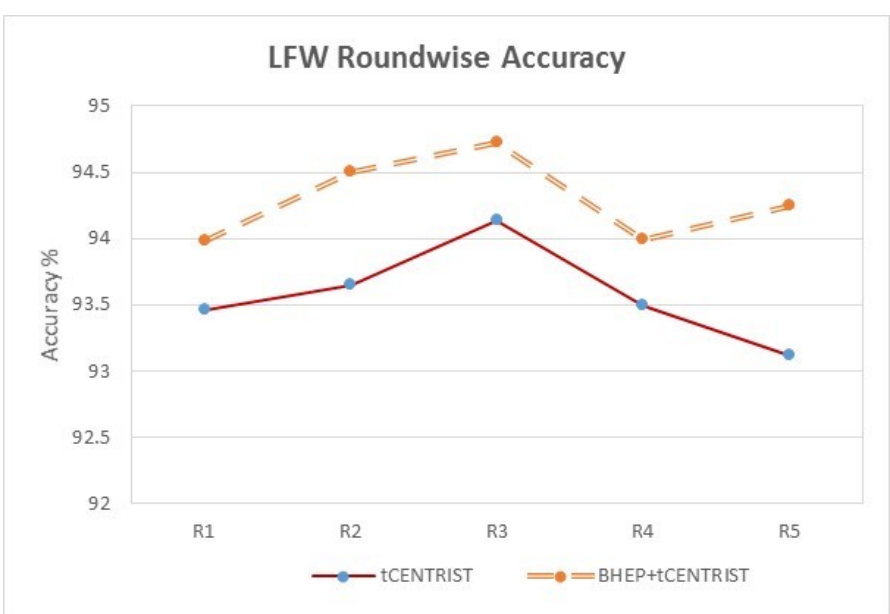

Fig. 6. Round wise accuracy in LFW dataset.

Table 8. Experimental results of different feature extraction method in LFW dataset.

\begin{tabular}{|c|c|}
\hline \multicolumn{2}{|c|}{ method in LFW dataset. } \\
\hline Method & Accuracy (\%) \\
\hline LBP & $82.21 \pm 1.05$ \\
\hline LTP & $83.73 \pm 0.59$ \\
\hline LGP & $79.71 \pm 0.83$ \\
\hline CENTRIST & $92.75 \pm 1.33$ \\
\hline tCENTRIST & $93.57 \pm 0.59$ \\
\hline tCENTRIST+BHEP & $94.29 \pm 0.60$ \\
\hline
\end{tabular}

traction improve the performance. We can notice that percentages of accuracy in LFW and Adience dataset are better than FERET dataset after use of BHEP. This is because, FERET dataset captured on controlled environment and it contain less illumination and inconsistent lighting problem. LFW and Adience dataset are real life dataset. BHEP corrected the images which were captured on low lighting condition, thus rate of accuracy increased in these two dataset.

\section{CONCLUSIONS}

In this paper we have proposed a framework for gender recognition in both constrained and unconstrained environment. We have used an image enhancement technique BHEP for preprocessing and feature extraction method tCENTRIST. Using three different dataset LFW, Adience and FERET we have shown that our proposed framework performs better than several state of the art

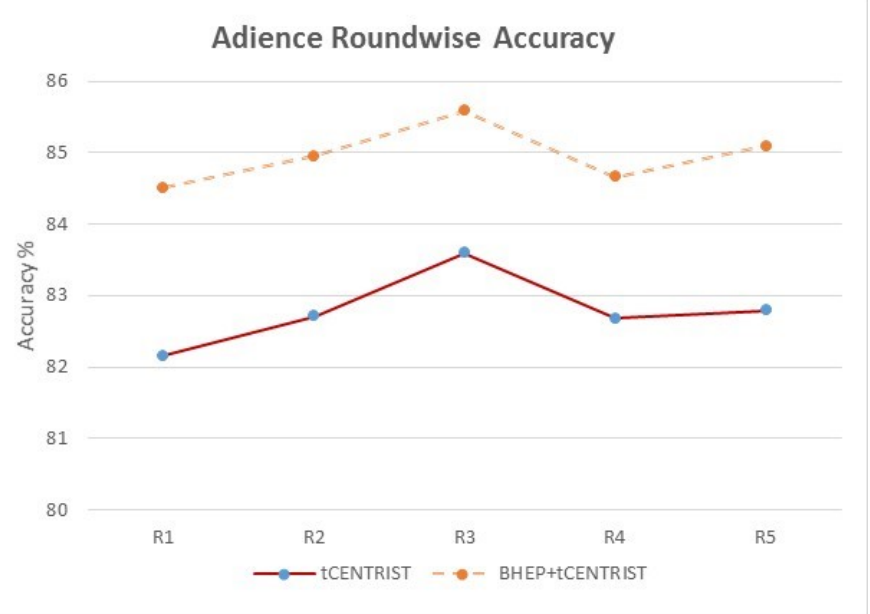

Fig. 7. Round wise accuracy in Adience Dataset.

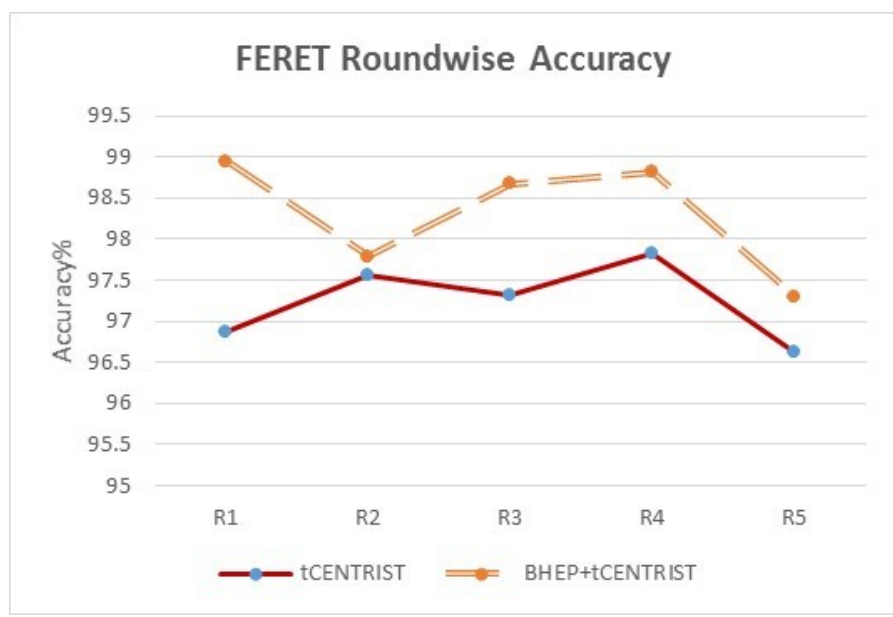

Fig. 8. Round wise accuracy in Color FERET Dataset.

method. Use of BHEP before feature extraction using tCENTRIST performs slightly better. This framework can also be used in several vision based classification such as object and scene classification. Performance of different feature extraction methods mainly degrades because of different angles of the face images of input 
Table 6. Performance of Proposed Gender Recognition Method without BHEP.

\begin{tabular}{|c|c|c|c|c|c|c|}
\hline & \multicolumn{2}{|c|}{ LFW without Preprocessing } & \multicolumn{2}{|c|}{ Adience DB without Preprocessing } & \multicolumn{2}{|c|}{ Color FERET without Preprocessing } \\
\hline & Female & Male & Female & Male & Female & Male \\
\hline Precision & 0.919847 & 0.946723 & 0.848466 & 0.818110 & 0.974411 & 0.971182 \\
\hline Recall & 0.810084 & 0.979512 & 0.869705 & 0.790476 & 0.9501 & 0.98538 \\
\hline F-measure & 0.861483 & 0.962839 & 0.858954 & 0.804051 & 0.962102 & 0.978229 \\
\hline
\end{tabular}

Table 7. Performance of Proposed Gender Recognition Method with BHEP.

\begin{tabular}{|c|c|c|c|c|c|c|}
\hline & \multicolumn{2}{|c|}{ LFW with Preprocessing } & \multicolumn{2}{|c|}{ Adience DB with Preprocessing } & \multicolumn{2}{c|}{ Color FERET with Preprocessing } \\
\cline { 2 - 6 } & Female & Male & Female & Male & Female & Male \\
\hline Precision & 0.923664 & 0.947666 & 0.859375 & 0.835580 & 0.986735 \\
Recall & 0.813445 & 0.980488 & 0.882542 & 0.805195 & 0.96507 & 0.979815 \\
F-measure & 0.865058 & 0.963798 & 0.870804 & 0.820106 & 0.975782 \\
\hline
\end{tabular}

\section{REFERENCES}

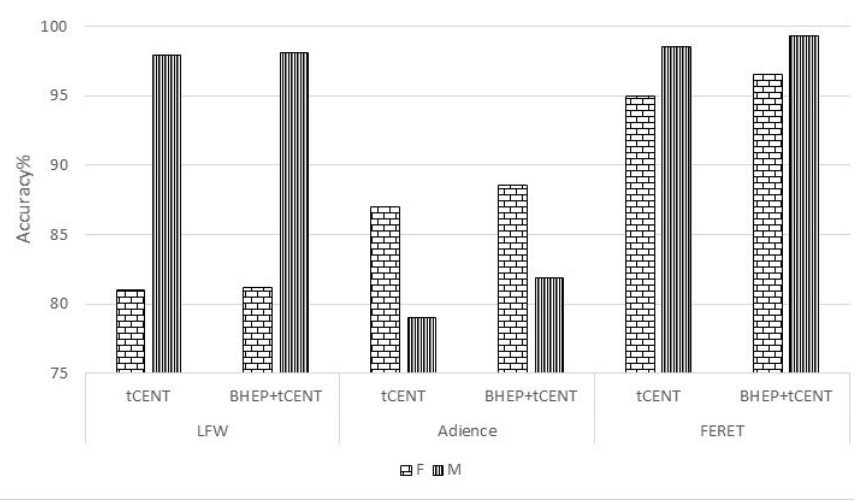

Fig. 9. Accuracy of proposed method in different dataset with and without BHEP.

Table 9. Experimental results of different feature extraction method in Adience dataset.

\begin{tabular}{|c|c|}
\hline Method & Accuracy (\%) \\
\hline LBP & $59.23 \pm 2.89$ \\
\hline LTP & $64.73 \pm 0.58$ \\
\hline LGP & $54.84 \pm 5.31$ \\
\hline CENTRIST & $82.49 \pm 0.89$ \\
\hline tCENTRIST & $82.79 \pm 0.55$ \\
\hline tCENTRIST+BHEP & $84.96 \pm 0.57$ \\
\hline
\end{tabular}

Table 10. Experimental results of different feature extraction method in Color FERET dataset.

\begin{tabular}{|c|c|}
\hline Method & Accuracy (\%) \\
\hline LBP & $83.58 \pm 2.16$ \\
\hline LTP & $80.30 \pm 1.95$ \\
\hline LGP & $76.09 \pm 0.53$ \\
\hline CENTRIST & $96.75 \pm 1.05$ \\
\hline tCENTRIST & $97.24 \pm 0.76$ \\
\hline tCENTRIST+BHEP & $98.30 \pm 1.01$ \\
\hline
\end{tabular}

datasets. A more sophisticated preprocessing method like by applying dynamic size blocks for feature extraction in input images to ameliorate the pose variation can be applied to improve the accuracies of current state of the art methods.
[1] Timo Ahonen, Abdenour Hadid, and Matti Pietikainen. Face description with local binary patterns: Application to face recognition. IEEE transactions on pattern analysis and machine intelligence, 28:2037-2041, 2006.

[2] Feroz Mahmud Amil, Md Mostafijur Rahman, Shanto Rahman, Emon Kumar Dey, and Mohammad Shoyaib. Bilateral histogram equalization with pre-processing for contrast enhancement. In Software Engineering, Artificial Intelligence, Networking and Parallel/Distributed Computing (SNPD), 2016 17th IEEE/ACIS International Conference on, pages 231-236. IEEE, 2016.

[3] Ramin Azarmehr, Robert Laganiere, Won-Sook Lee, Christina $\mathrm{Xu}$, and Daniel Laroche. Real-time embedded age and gender classification in unconstrained video. In Proceedings of the IEEE Conference on Computer Vision and Pattern Recognition Workshops, pages 57-65, 2015.

[4] Shumeet Baluja and Henry A Rowley. Boosting sex identification performance. International Journal of computer vision, 71(1):111-119, 2007.

[5] Emon Kumar Dey, Md Nurul Ahad Tawhid, and Mohammad Shoyaib. An automated system for garment texture design class identification. Computers, 4:265-282, 2015.

[6] Eran Eidinger, Roee Enbar, and Tal Hassner. Age and gender estimation of unfiltered faces. IEEE Transactions on Information Forensics and Security, 9:2170-2179, 2014.

[7] Beatrice A Golomb, David T Lawrence, and Terrence J Sejnowski. Sexnet: A neural network identifies sex from human faces. In NIPS, volume 1, page 2, 1990.

[8] Rafael C Gonzalez, Richard E Woods, et al. Digital image processing. Prentice hall Upper Saddle River:, NJ, 2002.

[9] Zhenhua Guo, Lei Zhang, and David Zhang. A completed modeling of local binary pattern operator for texture classification. IEEE Transactions on Image Processing, 19:1657$1663,2010$.

[10] Gary B Huang, Manu Ramesh, Tamara Berg, and Erik Learned-Miller. Labeled faces in the wild: A database for studying face recognition in unconstrained environments. Technical report, Technical Report $07-49$, University of Massachusetts, Amherst, 2007.

[11] Shih-Chia Huang, Fan-Chieh Cheng, and Yi-Sheng Chiu. Efficient contrast enhancement using adaptive gamma correction with weighting distribution. IEEE Transactions on Image Processing, 22:1032-1041, 2013. 
[12] Bongjin Jun, Inho Choi, and Daijin Kim. Local transform features and hybridization for accurate face and human detection. IEEE transactions on pattern analysis and machine intelligence, 35:1423-1436, 2013.

[13] Yeong-Taeg Kim. Contrast enhancement using brightness preserving bi-histogram equalization. IEEE transactions on Consumer Electronics, 43:1-8, 1997.

[14] Svetlana Lazebnik, Cordelia Schmid, and Jean Ponce. Beyond bags of features: Spatial pyramid matching for recognizing natural scene categories. In 2006 IEEE Computer Society Conference on Computer Vision and Pattern Recognition (CVPR'06), pages 2169-2178. IEEE, 2006.

[15] Gil Levi and Tal Hassner. Age and gender classification using convolutional neural networks. In Proceedings of the IEEE Conference on Computer Vision and Pattern Recognition Workshops, pages 34-42, 2015.

[16] Erno Makinen and Roope Raisamo. Evaluation of gender classification methods with automatically detected and aligned faces. IEEE Transactions on Pattern Analysis and Machine Intelligence, 30:541-547, 2008.

[17] Choon Boon Ng, Yong Haur Tay, and Bok Min Goi. Visionbased human gender recognition: A survey. arXiv preprint arXiv:1204.1611, 2012.

[18] Timo Ojala, Matti Pietikainen, and Topi Maenpaa. Multiresolution gray-scale and rotation invariant texture classification with local binary patterns. IEEE Transactions on pattern analysis and machine intelligence, 24:971-987, 2002.

[19] P Jonathon Phillips, Hyeonjoon Moon, Syed A Rizvi, and Patrick J Rauss. The feret evaluation methodology for facerecognition algorithms. IEEE Transactions on pattern analysis and machine intelligence, 22:1090-1104, 2000.

[20] P Jonathon Phillips, Harry Wechsler, Jeffery Huang, and Patrick J Rauss. The feret database and evaluation procedure for face-recognition algorithms. Image and vision computing, 16:295-306, 1998.

[21] M. M. Rahman, S. Rahman, M. Kamal, M. Abdullah-AlWadud, E. K. Dey, and M. Shoyaib. Noise adaptive binary pattern for face image analysis. In 2015 18th International Conference on Computer and Information Technology (ICCIT), pages 390-395, 2015.

[22] Md Mostafijur Rahman, Shanto Rahman, Emon Kumar Dey, and Mohammad Shoyaib. A gender recognition approach with an embedded preprocessing. International Journal of Information Technology and Computer Science (IJITCS), 7:19, 2015.

[23] Adín Ramírez Rivera, Jorge Rojas Castillo, and Oksam Chae. Local directional texture pattern image descriptor. Pattern Recognition Letters, 51:94-100, 2015.

[24] Gregory Shakhnarovich, Paul A Viola, and Baback Moghaddam. A unified learning framework for real time face detection and classification. In Automatic Face and Gesture Recognition, 2002. Proceedings. Fifth IEEE International Conference on, pages 14-21. IEEE, 2002.

[25] Caifeng Shan. Learning local binary patterns for gender classification on real-world face images. Pattern Recognition Letters, 33:431-437, 2012.

[26] Karen Simonyan, Omkar M Parkhi, Andrea Vedaldi, and Andrew Zisserman. Fisher vector faces in the wild. In $B M V C$, page 4, 2013.
[27] Xiaoyang Tan and Bill Triggs. Enhanced local texture feature sets for face recognition under difficult lighting conditions. IEEE transactions on image processing, 19:1635-1650, 2010.

[28] Yu Wang, Qian Chen, and Baeomin Zhang. Image enhancement based on equal area dualistic sub-image histogram equalization method. IEEE Transactions on Consumer Electronics, 45:68-75, 1999.

[29] Jianxin Wu and Jim M Rehg. Centrist: A visual descriptor for scene categorization. IEEE transactions on pattern analysis and machine intelligence, 33:1489-1501, 2011.

[30] Ramin Zabih and John Woodfill. Non-parametric local transforms for computing visual correspondence. In European conference on computer vision, pages 151-158. Springer, 1994.

[31] Baochang Zhang, Yongsheng Gao, Sanqiang Zhao, and Jianzhuang Liu. Local derivative pattern versus local binary pattern: face recognition with high-order local pattern descriptor. IEEE transactions on image processing, 19:533-544, 2010.

[32] Wenhao Zhang, Melvyn L Smith, Lyndon N Smith, and Abdul Farooq. Gender recognition from facial images: two or three dimensions? JOSA A, 33:333-344, 2016.

[33] Zhanpeng Zhang, Ping Luo, Chen Change Loy, and Xiaoou Tang. Facial landmark detection by deep multi-task learning. In European Conference on Computer Vision, pages 94-108. Springer, 2014. 